\title{
DISCOVERY OF POLARIZATION REVERBERATION IN NGC 4151
}

\author{
C. Martin Gaskell ${ }^{1,2,5}$, René W. Goosmann ${ }^{3}$, Nelly I. Merkulova ${ }^{4,6}$, \\ Nikolay M. ShaKhovsKoY ${ }^{4}$, AND Masatoshi ShoJI ${ }^{1,2}$ \\ ${ }^{1}$ Department of Physics \& Astronomy, University of Nebraska, Lincoln, NE 68588-0111, USA; martin.gaskell@uv.cl \\ 2 Astronomy Department, University of Texas, Austin, TX 78712-0259, USA; mshoji@ astro.as.utexas.edu \\ ${ }^{3}$ Observatoire astronomique de Strasbourg, 11 rue de l'Université, F-67000 Strasbourg, France; rene.goosmann@astro.unistra.fr \\ ${ }^{4}$ Crimean Astrophysical Observatory, Nauchny, Crimea 98409, Ukraine \\ ${ }^{5}$ Centro de Astrofísica de Valparaíso y Departamento de Física y Astronomía, Universidad de Valparaíso, Av. Gran Bretaña 1111, Valparaíso, Chile \\ Received 2011 June 13; accepted 2007 June 18; published 2012 April 3
}

\begin{abstract}
Observations of the optical polarization of NGC 4151 in 1997-2003 show variations of an order of magnitude in the polarized flux while the polarization position angle remains constant. The amplitude of variability of the polarized flux is comparable to the amplitude of variability of the total $U$-band flux, except that the polarized flux follows the total flux with a lag of $8 \pm 3$ days. The time lag and the constancy of the position angle strongly favor a scattering origin for the variable polarization rather than a non-thermal synchrotron origin. The orientation of the position angle of the polarized flux (parallel to the radio axis) and the size of the lag imply that the polarization arises from electron scattering in a flattened region within the low-ionization component of the broad-line region. Polarization from dust scattering in the equatorial torus is ruled out as the source of the lag in polarized flux because it would produce a larger lag and, unless the half-opening angle of the torus is $>53^{\circ}$, the polarization would be perpendicular to the radio axis. We note a long-term change in the percentage of polarization at similar total flux levels, and this could be due either to changing non-axisymmetry in the optical continuum emission or a change in the number of scatterers on a timescale of years.
\end{abstract}

Key words: galaxies: active - galaxies: individual (NGC 4151) - galaxies: Seyfert - polarization - quasars: general

\section{INTRODUCTION}

Shklovskii (1953) pointed out that synchrotron radiation should be highly polarized, and the detection in 1953 of polarization of the Crab Nebula demonstrated the synchrotron nature of its continuum radiation (Dombrovskii 1958). It was therefore natural to search for polarization of the optical emission of Seyfert galaxies (Dibai \& Shakhovskoy 1966). Optical polarization of NGC 4151 was discovered by Babadzhanyants \& Hagen-Thorn (1969) who also reported that the polarization was variable. The polarization was studied in more detail by Kruszewski (1971) who found that the polarization in the $V$ band varied by a factor of four over a nine-month period. The detection of variable polarization was taken as evidence of a synchrotron origin of the continuum.

The discovery of polarization of emission lines (Angel et al. 1976) in NGC 1068 showed that the polarization of type-2 active galactic nuclei (AGNs) was due to scattering and not synchrotron emission. The origin of the optical polarization in type-1 AGNs, however, has remained ambiguous. Stockman et al. (1979) discovered that the optical polarization of radioloud AGNs was parallel to the radio jet axis. They considered this to be either the result of intrinsic polarization of optical synchrotron emission or the result of scattering from a flattened distribution of scatters. Subsequently, Antonucci $(1982,1983)$ showed that, in general, polarization tends to be parallel to the radio axis in type-1 AGNs and perpendicular in type-2 AGNs. Since the polarization of broad lines is roughly similar to the polarization of the continuum (Goodrich \& Miller 1994), this showed that the polarization was due to scattering. It was shown by Smith et al. (2004) and Goosmann \& Gaskell (2007) that type-1 polarization can be explained by a flattened equatorial

\footnotetext{
6 Deceased: 2004 December 12.
}

scattering region. Nonetheless because of the variability of optical polarization it has often been assumed, by analogy with blazars (where there is no doubt that the optical polarization variations have a non-thermal origin), that synchrotron radiation also contributes to optical polarization in non-blazar AGNs (e.g., Giannuzzo \& Salvati 1993). In this paper, we attempt to resolve the question of the nature of the optical polarization by investigating the relationship between the variability of the polarized flux and the total flux.

\section{OBSERVATIONS}

NGC 4151 was observed through a 15 arcsec diameter aperture with the UBVRI Double Image Chopping PhotometerPolarimeter (Piirola 1988) on the $1.25 \mathrm{~m}$ AZT-11 telescope of the Crimean Astrophysical Observatory. The polarimeter gives simultaneous measurement of the linear polarization in the standard Johnson bands by using dichroic filters. The resulting passbands have effective wavelengths of $3600,4400,5400,6900$, and $8300 \AA$ which are close to the standard Johnson UBRVI system. Observations of NGC 4151 were made on 58 nights between 1997 May and 2003 May. About 30 separate measurements were made per night. Errors in the flux and polarized flux were estimated by comparing measurements taken within \pm 5 nights of each other.

\section{RESULTS}

Merkulova \& Shakhovskoy (2006) have already discussed the variability of the total flux and polarized flux in the five passbands. As is normal when there is substantial host galaxy contamination because of the use of a large photometric aperture, the highest amplitude total flux variability is in the $U$ band. This is shown in Figure 5 of Merkulova (2002). In NGC 4151, as in other AGNs, the $B$-band and $U$-band fluxes vary 


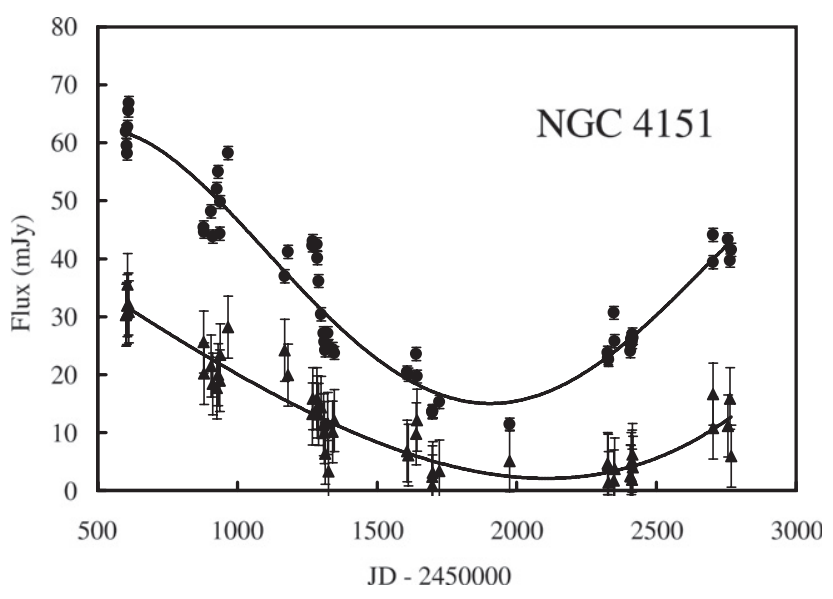

Figure 1. Variations in total $U$-band flux (top curve) and $B$-band polarized flux (lower curve). The polarized flux has been multiplied by a factor of 30 for plotting convenience. The polarization position angles in both wave bands are consistent with each other and remained nearly constant at $92^{\circ} \pm 1.6$ during the whole observational campaign. The two curves are fourth-order polynomial fits through the data.

essentially simultaneously. Crenshaw et al. (1996) found that the UV continuum variations in NGC 4151 are simultaneous to within $\sim \pm 0.15$ days across the UV, and Edelson et al. (1996) get an upper limit of $\sim 1$ day to the lag of the $\lambda 5100$ continuum with respect to the continuum at $\lambda 1275$ (see Figure 10 of Lyuty 2005). Wavelength-dependent lags are seen at longer wavelengths in the $R$ and $I$ bands (Lyuty 2005; Sergeev et al. 2005), but Gaskell (2007) argues that these are due to contamination by emission from hot dust. We do not expect a significant lag of the $B$ band relative to the $U$ band and there is no observational evidence for one. Oknyanskij et al. (2003) find the $V$ band to be lagging behind the $B$ band by $+0.2 \pm 0.4$ days, and Sergeev et al. (2005) find it to be lagging by $-0.07 \pm 0.22$ days. The last two limits are important because the former overlaps the first third of our polarimetric monitoring and the later the last third. We can thus be confident that there is no significant delay between the $U$ and $B$ bands and that variations in $U$ are an excellent indicator of variations in $B$.

The polarization was variable in all five passbands and it too might be expected to be highest toward shorter wavelengths, but Merkulova \& Shakhovskoy (2006) find that both the polarization and polarization variability of NGC 4151 peak in the $B$ band and show a significant drop in the $U$ band. This is found in other objects. Kishimoto et al. (2004) find from their spectropolarimetry that the polarized flux spectrum of AGNs commonly peaks in the region of the $B$ band and drops off in the $U$ band. They attribute this to a Balmer absorption edge in the accretion-disk emission. Detailed modeling shows that the spectrophotometry of the Balmer edge region is well fit with a Doppler-broadened Balmer absorption edge (Gaskell 2009b).

Because the polarization variability of NGC 4151 is at a maximum in the $B$ band, and because the signal-to-noise ratio is also highest in the $B$ band (because of the amplitude of the variability and the high quantum efficiency of the detector in the $B$ band), we only consider the variability of the $B$-band polarization here. We show the variability of the $B$-band polarized flux and the variability of the total flux (taken from the $U$ band) in Figure 1.

As can be seen from Figure 1, during the period 1997-2003 the $U$-band flux showed long-term variability of at least a factor of four, and the $B$-band polarized flux showed long-term variability of a factor of 10. Merkulova \& Shakhovskoy (2006)

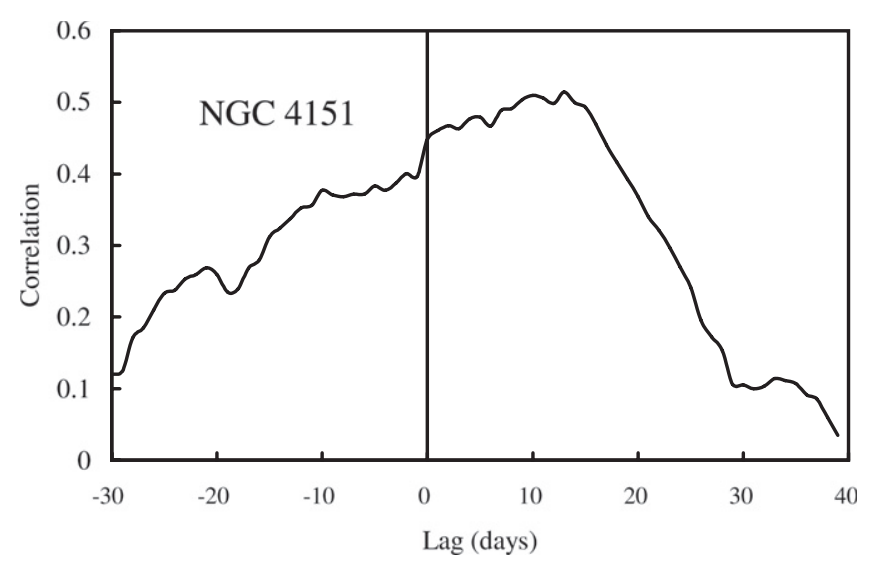

Figure 2. Cross-correlation function for the polarized flux and total $B$-band flux for the entire six-year data set. A positive lag corresponds to the polarized flux following the total flux.

find that the position angle (P.A.) of the variable polarization remained nearly constant at $92^{\circ} \pm 1.6$ which is consistent with earlier measurements (Antonucci 1983; Martel 1998) and with being parallel to the radio axis (Antonucci 1983).

\section{ANALYSIS}

\subsection{Long-term Variability}

During the period 1997-2003 NGC 4151 declined from the end of a high state and passed through a low minimum level of activity (Lyuty 2006). There is some evidence for a slow longterm change in the degree of polarization over the seven-year period. This is most obvious if we compare years where the mean $U$-band continuum level was approximately the same. As can be seen from Figure 1, the polarized flux around MJD 2400 is more than a factor of two lower than that around MJD 1300 when the total fluxes are comparable. Similarly, the polarized flux is also lower around MJD 2750 compared with MJD 1200. This change in the percentage polarization after the photometric minimum around MJD 2000 suggests a possible reduction on a dynamical timescale in the number of scatterers after the low state. Another possible explanation, strongly favored by other observations (see Gaskell 2010, 2011 for extensive discussion), is that the continuum variability could be non-axisymmetric and the anisotropy could be varying from year to year. Unfortunately, we did not have good polarimetric coverage in the year of the photometric minimum.

\subsection{Short-term Variability}

In order to search for polarization reverberation on short timescales, we have removed the long-term trends by subtracting fourth-order polynomials (shown in Figure 1) from the total flux and polarized flux time series. The two time series were crosscorrelated using the interpolation method of Gaskell \& Sparke (1986). Details of the method are given in Gaskell \& Peterson (1987). We show the resulting cross-correlation function (CCF) for the entire data set in Figure 2.

The centroid of the CCF calculated from the first moments above 0.7 times the peak correlation gives a lag of 8 days. In Table 1, we also give the considerably less certain lags from the centroids of the CCFs for the individual observing seasons. The median of the lags for the individual years is consistent with the lag from the whole of the seven observing seasons. The differences in lags in Table 1 are not statistically significant, but if the variability is non-axisymmetric (Gaskell 2010, 2011), 
Table 1

Centroids of Lags for Individual Years

\begin{tabular}{lcc}
\hline \hline Year & $\begin{array}{c}\text { Lag } \\
\text { (days) }\end{array}$ & Error \\
\hline 1997 & 7 & \pm 4 \\
1998 & 43 & \pm 6 \\
1999 & 7 & \pm 7 \\
2000 & 10 & \pm 5 \\
2001 & $\ldots$ & \\
2002 & 14 & \pm 8 \\
2003 & 7 & \pm 5 \\
All years & 8 & \\
\hline
\end{tabular}

future better observations could show that real variations in the polarization lags vary from year to year.

As is well known (Gaskell \& Peterson 1987), the distribution of errors in the peaks in CCFs is non-Gaussian with a tail extending to high errors. This can be seen in the simulations of Maoz \& Netzer (1989), White \& Peterson (1994), and Peterson et al. (1998). We estimated the error in the lag of the polarized flux in three different ways. First, we used the formula of Gaskell $\&$ Peterson (1987). This gave an error of \pm 3.5 days for the lag derived from the whole data set. We also performed Monte Carlo simulations where we added noise to the observations equal to the observational errors. This gave an error in the lag of \pm 2.5 days. Finally, where possible we calculated the lags for the individual years as described above. They are again the first moment lags calculated above 0.7 of the peak in the CCF. These lags are shown in Table 1 with the approximate errors from the Gaskell \& Peterson (1987) formula. As can be seen, even though the errors are substantially larger because of the small number of observations, the median lag for the individual years ( 8.5 days) is close to the lag we obtained from all years. The median of the absolute values of the differences of the individual lags for each year from the lag for all years is 1.5 days. These three separate error estimates suggest that the error in the lag for the whole sample is of the order of \pm 3 days.

\section{DISCUSSION}

An $\sim 8$ day lag of the polarized flux has no natural explanation if the polarization has a synchrotron origin. However, a lag can naturally be explained as a light-travel-time delay of scattered radiation (Giannuzzo \& Salvati 1993). As noted above, scattering off both electrons and dust has long been considered as a cause of polarization in AGNs. Electron scattering could take place in clouds along the axis of symmetry or in a flattened equatorial distribution of electrons (Smith et al. 2004, 2005). Dust scattering in the torus will also be responsible for polarization. To investigate which process dominates in producing the polarized flux, we modeled polarization reverberation in NGC 4151 with the Monte Carlo radiative transfer code STOKES (Goosmann \& Gaskell 2007; Goosmann et al. 2007). In order to model polarization variability, we modified STOKES to time-tag each photon going through the program.

\subsection{Polarization from the Dusty Torus}

For the period 1990-1998, Oknyanskij et al. (1999) find a delay of $35 \pm 8$ days for the $K$ band relative to the $U$ band. This includes the peak of activity at the start of our polarimetric monitoring. For the low state during our monitoring, Minezaki et al. (2004) find a similar delay of $48 \pm 2.5$ days, and by the end of our observations Swain et al. (2003) constrained the radial extension of the $K$-band emitting region to $0.04 \mathrm{pc}$, which also corresponds to a time lag of $\sim 48$ days. Kishimoto et al. (2009) found an identical $K$-band size in 2009. Note that Swain et al. (2003) believed that the $K$-band emission comes from the outer accretion disk while Kishimoto et al. (2007, 2009) attribute it to the inner boundary of the torus. The difference in both interpretations may lie in the assumed dust sublimation radius. If one assumes a grain-size distribution favoring larger grains, the dust can survive at a relatively close distance of $0.04 \mathrm{pc}$ from the central source. Indications that dust grains in AGNs tend to be larger than in standard Milky Way dust are found from examining quasar reddening curves (Gaskell et al. 2004).

Oknyanskij (1993) did determine a $K$-band lag during the low state of NGC 4151 in the 1970s of $18 \pm 6$ days which is marginally consistent with the polarization reverberation delay we measure, but as Oknyanskij et al. (2006) point out, their observations and those of Minezaki et al. (2004) show that, as would be expected, the high state of NGC 4151 at the start of our polarimetric monitoring destroyed the dust close to the black hole, and the $K$-band observations show that the dust had still not reappeared at smaller radii within several years of the high state.

Overall, there is good observational evidence that the inner radius of the torus did not change significantly during our monitoring that finished in 2003 May. Koshida et al. (2009) report that the inner boundary of the torus moved in after 2003, but when Pott et al. (2010) and Kishimoto et al. (2011) took new interferometry data, the radius of the $K$-band emission region was again at $\sim 0.04 \mathrm{pc}$ from the center. The $K$-band emission comes from the hottest dust, and lags given by the cross-correlation method are biased toward material at the smallest radii (Gaskell $\&$ Sparke 1986) so the $K$-band lag is giving the inner radius of the dust torus. From the Oknyanskij et al. (1999) and Minezaki et al. (2004) measurements, we can conclude the inner radius of the dusty torus during our polarimetric monitoring was $\sim 40$ lt-day. This strongly rules out dust scattering being the cause of the polarization reverberation we detect.

We modeled dust scattering off a variety of optically thick torus geometries. We considered cylindrical tori and tori with elliptical cross sections. In all cases, we set the inner radii to be 40 lt-day as indicated by the IR reverberation mapping. Various opening and viewing angles were modeled. The resulting first moment delays in the polarized flux were $\sim 43$ days (i.e., slightly greater than the inner radius), and varied only by $10 \%-15 \%$ with changing geometries and viewing angles. These modeled lags are clearly inconsistent with the $\sim 8$ days lag we find from the observations. So long as the torus was not too thin (i.e., the half-opening angle is not $>53^{\circ}$ ), the polarization of the dust model averaged over the lag was perpendicular to the radio axis for all the type-1 viewing angles, which is inconsistent with the observed P.A. We thus believe that scattering from the torus is not the cause of the lag in the polarized flux. For more extensive discussion of the effect of the geometry and viewing angle on the degree and direction of polarization, see Goosmann \& Gaskell (2007).

\subsection{Polarization from a Flattened Electron-scattering Region}

Scattering off a polar distribution of electrons (Giannuzzo \& Salvati 1993) also produces the wrong polarization angle, but a flattened electron distribution produces polarization parallel to the radio axis (see discussion in Goosmann \& Gaskell 2007). We therefore used STOKES to model the polarization lag from cylindrical electron-scattering disks. We found that for disks which were optically thick to electron scattering, the lag was 
equal to the inner radius. Thus, if the electron-scattering region is optically thick in NGC 4151, it has an inner radius of $\sim 8$ lt-day.

The geometry of the scattering region is similar to that of a broad-line region (BLR; see a review by Gaskell 2009a). Furthermore, the lag we find is comparable to the size of the BLR of NGC 4151. Gaskell \& Sparke (1986) obtained a size of $5 \pm 2$ lt-day for the radius of the $\mathrm{C}$ IV emitting region of the BLR in NGC 4151 during 1978-1980. Metzroth et al. (2006) obtained radii of $3.4 \pm 1.3$ days for 1988 and $3.3 \pm 0.9$ days for 1991 . They also obtained identical radii for He II. Gaskell \& Sparke (1986) estimated the size of the region emitting $\mathrm{H} \beta$ and $\mathrm{H} \gamma$ to be $\sim 6$ lt-day during 1980-1981. More recent observations by Bentz et al. (2006) give $6.6 \pm 1$ lt-day for 2005 . Note again that the responsivity-weighted radii given by reverberation mapping are biased toward the inner radii of the emitting region.

While our observed polarization lag is in good agreement with the observed radii of the $\mathrm{C}$ IV and $\mathrm{H} \beta$ emission in NGC 4151, the observed inner radius for a given line reflects the radial variation of ionization and there is almost certainly BLR gas inside that radius. If the gas density of the electron-scattering region in our STOKES models is somewhat lower than a canonical BLR density (i.e., $10^{8} \mathrm{~cm}^{-3}$ rather than $10^{10} \mathrm{~cm}^{-3}$ ), then the model is no longer optically thick, the mean free path to electron scattering becomes significant, and we find that the lag is greater than the inner radius of our hypothetical disk. For example, a disk with a density of $10^{8} \mathrm{~cm}^{-3}$ would give a mean free path of $\sim 6$ lt-day. It is therefore possible to reproduce the observed polarization lag without having to have an artificial hole in the middle of the distribution of electrons.

Interestingly, the radius we obtain for the electron-scattering disk (i.e., toward the outer edge of the BLR) is in good agreement with the location deduced quite independently by Smith et al. (2005) from modeling the change in polarization with velocity across the profiles of broad emission lines. Nevertheless, it is more intuitive to assume a continuous accretion flow from the torus down to the supermassive black hole.

\section{CONCLUSIONS}

The polarized flux of NGC 4151 appears to lag the unpolarized flux by $\sim 8$ days which is comparable to the light-crossing time of the BLR. We interpret this as the result of the extra lighttravel time of the scattered photons. Dust in the torus is ruled out as the source of polarization by the angle of polarization and because the observed lag is too short. Both the direction of polarization and the lag can naturally be explained instead by electron scattering in a flattened region of similar size to the low-ionization BLR. We have also detected a possible change in the percentage polarization of NGC 4151 at similar flux levels on a timescale of years, and we attribute this to either longterm changes in the number of scattering electrons or variable asymmetric emission in the disk.

It is clearly important to confirm these results with better data. We believe that further polarimetric reverberation mapping of AGNs (especially spectropolarimetric reverberation mapping) should be a powerful technique for distinguishing between models of the structure of the inner regions of AGNs.

We are grateful to Makoto Kishimoto for helpful comments. This research has been supported by the U.S. National Science Foundation through grants AST 03-07912 and AST 08-03883, the University of Nebraska UCARE program, the GEMINICONICYT Fund of Chile through project $N^{\circ} 32070017$, and the French $G d R$ PCHE. Polarimetric observations at the Crimean
Astrophysical Observatory were made possible in part by Award No.UP1-2549-CR-03 of the U.S. Civilian Research \& Development Foundation (CRDF).

\section{REFERENCES}

Angel, J. R. P., Stockman, H. S., Woolf, N. J., Beaver, E. A., \& Martin, P. G. 1976, ApJ, 206, L5

Antonucci, R. R. J. 1982, Nature, 299, 605

Antonucci, R. R. J. 1983, Nature, 303, 158

Babadzhanyants, M. K., \& Hagen-Thorn, V. A. 1969, Astron. Tsirk., 526, 1

Bentz, M. C., Denney, K. D., Cackett, E. M., et al. 2006, ApJ, 651, 775

Crenshaw, D. M., Rodriguez-Pascual, P. M., Penton, S. V., et al. 1996, ApJ, 470, 322

Dibai, E. A., \& Shakhovskoy, N. M. 1966, Astron. Tsirk., 375, 1

Dombrovskii, V. A. 1958, AZh, 35, 687 (Engl. Trasl. SvA, 2, 646)

Edelson, R. A., Alexander, T., Crenshaw, D. M., et al. 1996, ApJ, 470, 364

Gaskell, C. M. 2007, in ASP Conf. Ser. 373, The Central Engine of Active Galactic Nuclei, ed. L. C. Ho \& J.-M. Wang (San Francisco, CA: ASP), 596

Gaskell, C. M. 2009a, New Astron. Rev., 53, 140

Gaskell, C. M. 2009b, BAAS, 41, 477

Gaskell, C. M. 2010, ApJ, submitted (arXiv:1008.1057)

Gaskell, C. M. 2011, Balt. Astron., 20, 392

Gaskell, C. M., Goosmann, R. W., Antonucci, R. R. J., \& Whysong, D. H. 2004, ApJ, 616, 147

Gaskell, C. M., \& Peterson, B. M. 1987, ApJS, 65, 1

Gaskell, C. M., \& Sparke, L. S. 1986, ApJ, 305, 175

Giannuzzo, E., \& Salvati, M. 1993, A\&A, 272, 411

Goodrich, R. W., \& Miller, J. S. 1994, ApJ, 434, 82

Goosmann, R. W., \& Gaskell, C. M. 2007, A\&A, 465, 129

Goosmann, R. W., Gaskell, C. M., \& Shoji, M. 2007, in IAU Symp. 238, Black Holes from Stars to Galaxies: Across the Range of Masses, ed. V. Karas \& G. Matt (Cambridge: Cambridge Univ. Press), 375

Kishimoto, M., Antonucci, R., Boisson, C., \& Blaes, O. 2004, MNRAS, 354, 1065

Kishimoto, M., Hönig, S. F., Antonucci, R., et al. 2009, A\&A, 507, L57

Kishimoto, M., Hönig, S. F., Antonucci, R., et al. 2011, A\&A, 527, A121

Kishimoto, M., Hönig, S. F., Beckert, T., \& Weigelt, G. 2007, A\&A, 476, 713

Koshida, S., Yoshii, Y., Kobayashi, Y., et al. 2009, ApJ, 700, L109

Kruszewski, A. 1971, Acta Astron., 21, 311

Lyuty, V. M. 2005, Astron. Lett., 31, 645

Lyuty, V. M. 2006, in ASP Conf. Ser. 360, AGN Variability from X-Rays to Radio Waves, ed. C. M. Gaskell, I. M. McHardy, B. M. Peterson, \& S. G. Sergeev (San Francisco, CA: ASP), 3

Maoz, D., \& Netzer, H. 1989, MNRAS, 236, 21

Martel, A. R. 1998, ApJ, 508, 657

Merkulova, N. I. 2002, A\&A, 387, 40

Merkulova, N. I., \& Shakhovskoy, N. M. 2006, in ASP Conf. Ser. 360, AGN Variability from X-Rays to Radio Waves, ed. C. M. Gaskell, I. M. McHardy, B. M. Peterson, \& S. G. Sergeev (San Francisco, CA: ASP), 65

Metzroth, K. G., Onken, C. A., \& Peterson, B. M. 2006, ApJ, 647, 901

Minezaki, T., Yoshii, Y., Kobayashi, Y., et al. 2004, ApJ, 600, L35

Oknyanskij, V. L. 1993, Astron. Lett., 19, 416

Oknyanskij, V. L., Horne., K., Lyutyi, V. M., et al. 2003, in ASP Conf. Ser. 290, Active Galactic Nuclei: From Central Engine to Host Galaxy, ed. S. Collin, F. Combes, \& I. Shlosman (San Francisco, CA: ASP), 119

Oknyanskij, V. L., Lyuty, V. M., Taranova, O. G., \& Shenavrin, V. I. 1999, Astron. Lett., 25, 483

Oknyanskij, V. L., Lyuty, V. M., Taranova, O. G., \& Shenavrin, V. I. 2006, in ASP Conf. Ser. 360, AGN Variability from X-Rays to Radio Waves, ed. C. M. Gaskell, I. M. McHardy, B. M. Peterson, \& S. G. Sergeev (San Francisco, CA: ASP), 75

Peterson, B. M., Wanders, I., Horne, K., et al. 1998, PASP, 110, 660

Piirola, V. 1988, in Polarized Radiation of Circumstellar Origin (Tucson, AZ: Vatican Observatory/Univ. Arizona Press), 735

Pott, J.-U., Malkan, M. A., Elitzur, M., et al. 2010, ApJ, 715, 736

Sergeev, S. G., Doroshenko, V. T., Golubinskiy, Yu. V., Merkulova, N. I., \& Sergeeva, E. A. 2005, ApJ, 622, 129

Shklovskii, I. S. 1953, in A Source Book in Astronomy \& Astrophysics, ed. K. R. Lang \& O. Gingerich (Cambridge, MA: Harvard Univ. Press), 488

Smith, J. E., Robinson, A., Alexander, D. M., et al. 2004, MNRAS, 350, 140

Smith, J. E., Robinson, A., Young, S., Axon, D. J., \& Corbett, E. A. 2005, MNRAS, 359, 846

Stockman, H. S., Angel, J. R. P., \& Miley, G. K. 1979, ApJ, 227, L55

Swain, M., Vasisht, G., Akeson, R., et al. 2003, ApJ, 596, L163

White, R. J., \& Peterson, B. M. 1994, PASP, 106, 879 